\title{
Understanding Behavior of Self-Regenerative Catalysts by Transmission Electron Microscopy and Density Functional Theory
}

\author{
X.Q. Pan ${ }^{1}$, M.B. Katz ${ }^{1}$, B.H. Li ${ }^{1}$, X.F. Du ${ }^{1}$, K. Zhang ${ }^{1}$, Y.W. Duan ${ }^{1}$, L.F. Allard ${ }^{2}$, L. Chen ${ }^{3}$, A. Van der \\ Ven $^{1}$ and G.W. Graham ${ }^{1}$ \\ ${ }^{1}$ Department of Materials Science and Engineering, University of Michigan, Ann Arbor, MI, USA \\ ${ }^{2 .}$ Materials Science \& Technology Division, Oak Ridge National Laboratory, Oak Ridge, TN, USA \\ ${ }^{3}$.Functional Materials and Nanodevices Division, Ningbo Institute of Materials Technology and \\ Engineering, Ningbo, Zhejiang, PRC
}

First promoted by Daihatsu Motor Co. as an 'intelligent catalyst' [1], the 'self-regenerative catalyst' is an automotive catalyst in which the precious metal (e.g. Pt, $\mathrm{Pd}, \mathrm{Rh})$ supposedly dissolves into a carefully chosen perovskite oxide support upon oxidation, and re-disperses as metallic particles upon reduction; cyclical redox conditions that are already present in gasoline engine exhaust due to the fuel injection control system. This is counter to the behavior of metal particles on most supports, that irreversibly coarsen during redox cycling. Though the mechanistics of such a system are of great interest to the catalyst community, the authors of the original report (and subsequent studies) utilized only bulk characterization techniques (e.g. EXAFS, XANES) to analyze the average structure, and thus they did not satisfactorily probe the local environment of the metal particles and/or ions.

Our recent studies of two prototypical self-regenerative catalyst systems, utilizing model thin-film catalysts examined by aberration-corrected scanning transmission electron microscopy (STEM), revealed a much more complex interplay between the metal and support than had been previously realized. For both $\mathrm{Pd} / \mathrm{LaFeO}_{3}$ and $\mathrm{Pt} / \mathrm{CaTiO}_{3}$, the dissolution and extrusion of metal upon redox cycling is significantly less facile than was reported. In the $\mathrm{Pd} / \mathrm{LaFeO}_{3}$ system, $\mathrm{Pd}$ particles on the surface do not dissolve into the support upon oxidation, but rather displace the oxide in their immediate vicinity and form hemispherical pits in which they are mechanically hindered from migration [2]. In the $\mathrm{Pt} / \mathrm{CaTiO}_{3}$ system, Pt can extrude from the oxide and re-dissolve, but it does so within the bulk of the support, rather than at the surface, leaving the metal particles unavailable for gas catalysis in most morphologies [3].

Further effort to understand the mechanistics of these systems has led to two complementary sets of studies. In one set we used advanced in situ STEM gas-reaction capabilities to study the redox reaction dynamics at conditions that mimic the ex situ studies conducted on the thin films. The reaction in the Ptbased system proved to be asymmetric, with metal extruding from the oxide within several seconds upon reduction, but dissolving back into the support only with hours of aggressive oxidation.

We also used density functional theory (DFT) to further understand the systems at a more fundamental thermodynamic level. In the case of $\mathrm{Pd} / \mathrm{LaFeO}_{3}, \mathrm{DFT}$ modeling shows that in heavily oxidizing conditions, $\mathrm{Pd}$ will interact strongly with $\mathrm{FeO}_{2}^{-}$-terminated $\mathrm{LaFeO}_{3}$, intermixing with the layer and displacing the material around it [4]. For $\mathrm{Pt} / \mathrm{CaTiO}_{3}$, phase diagrams were calculated under various oxygen pressures in order to fully understand the stabilities of the various phases in the system. From the phase diagrams, we can understand the thermodynamic origin of the cyclability of the selfregenerative catalyst [5]. 
References:

[1] Y. Nishihata et al., Nature. 418 (2002) p. 164.

[2] M.B. Katz et al., J. Amer. Chem. Soc. 133 (2011) p. 18090.

[3] M.B. Katz et al., J. Catal. 293 (2012) p. 145.

[4] B.H. Li et al., under review

[5] This research was supported by the National Science Foundation under grants DMR-0723032, DMR-0907191, and CBET-1159240, by Ford Motor Company under a Ford-UM Innovation Alliance grant, and by Protochips, Inc. Work at Oak Ridge National Laboratory was sponsored by the U.S. Department of Energy, Off. of Energy Efficiency \& Renewable Energy, Vehicle Technologies Program.

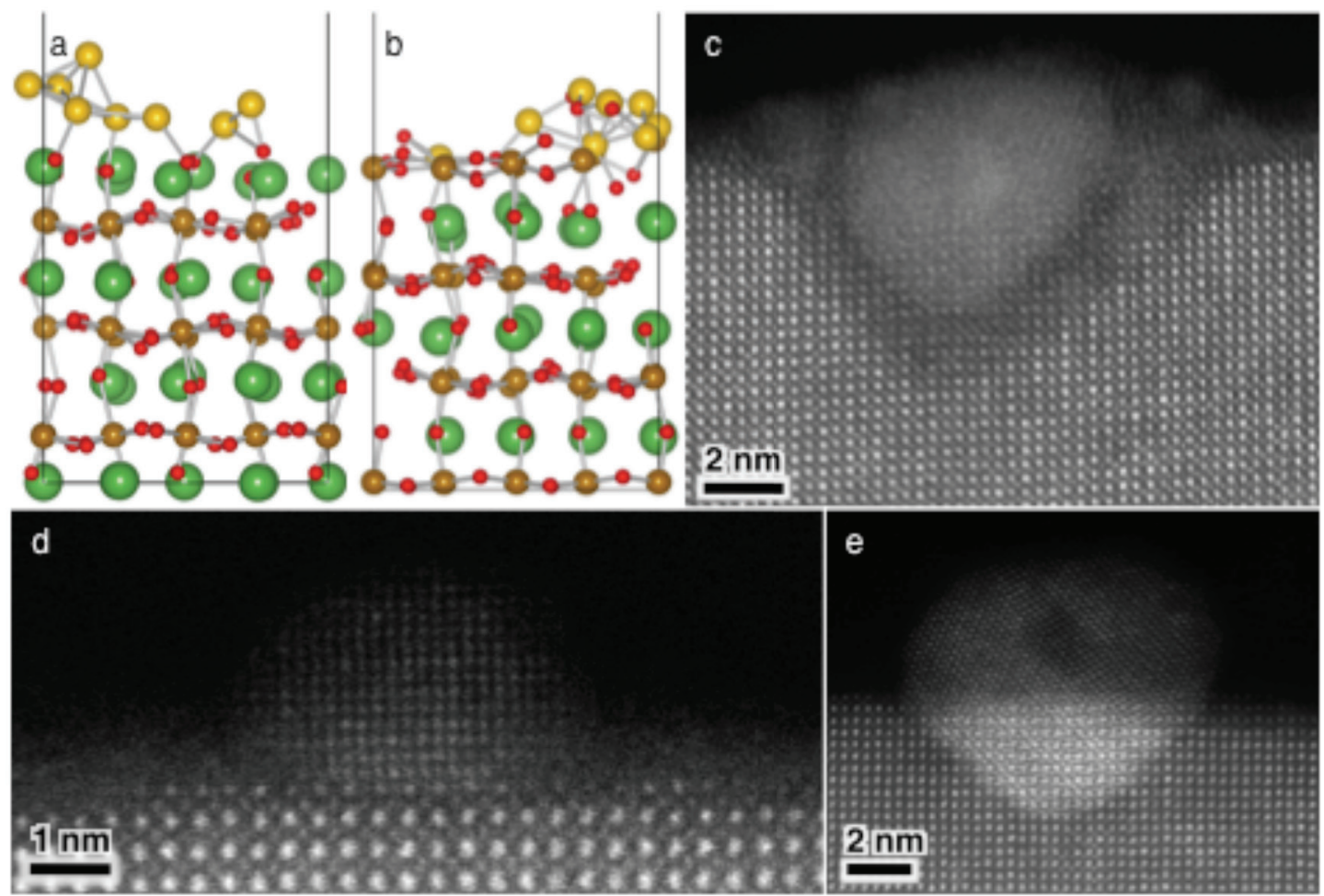

Figure 1. Simulations of a relaxed $\mathrm{Pd}$ cluster on the (a) $\mathrm{LaO}^{+}-$and (b) $\mathrm{FeO}^{-}$-terminated surfaces of $\mathrm{LaFeO}_{3}$ and STEM images of Pd particles on $\mathrm{LaFeO}_{3}$ films after (c) oxidation, (d) reduction, and (e) redox cycling.
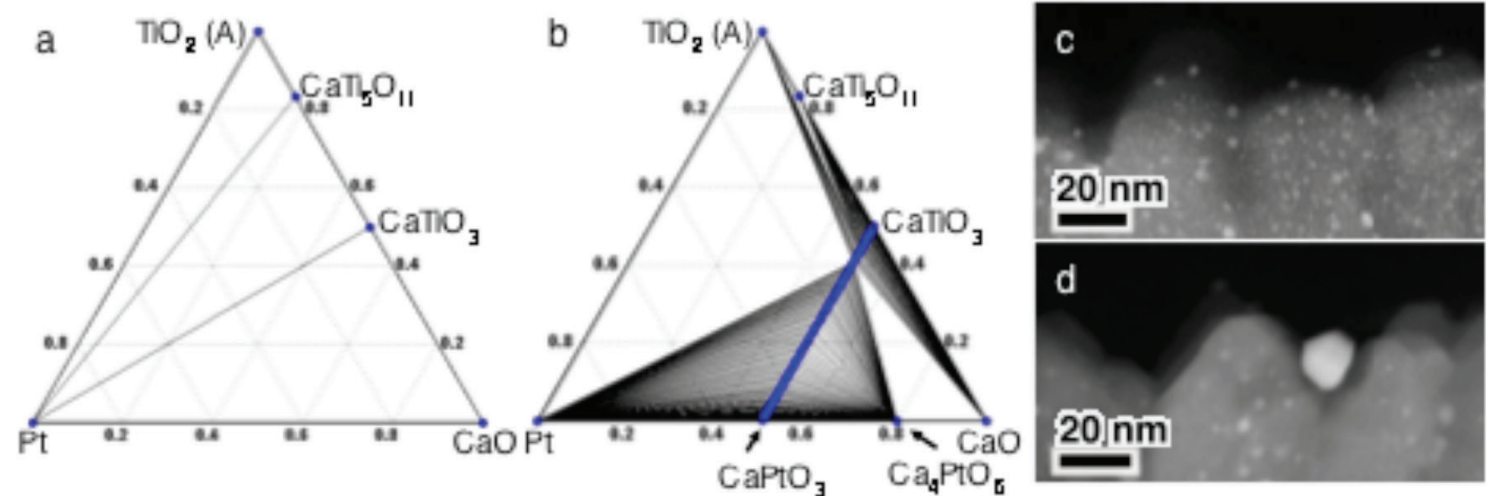

Figure 2. Calculated pseudo-ternary phase diagrams for the $\mathrm{CaO}_{\mathrm{x}}-\mathrm{TiO}_{\mathrm{y}}-\mathrm{PtO}_{\mathrm{z}}$ at $800{ }^{\circ} \mathrm{C}$ for (a) reducing and (b) oxidizing conditions, and STEM images of a $\mathrm{CaTi}_{0.95} \mathrm{Pt}_{0.05} \mathrm{O}_{3}$ thin film (nominal composition) after (c) reduction and (d) re-oxidation. 